\title{
CONVEX AND WEAKLY CONVEX DOMINATION IN PRISM GRAPHS
}

\author{
MONIKA ROSICKA \\ Faculty of Mathematics, Physics and Informatics \\ University of Gdańsk, 80-952 Gdańsk, Poland \\ Institute of Theoretical Physics and Astrophysics and \\ National Quantum Information Centre in Gdańsk \\ 81-824 Sopot, Poland \\ e-mail: mrosicka@inf.ug.edu.pl
}

\begin{abstract}
For a given graph $G=(V, E)$ and permutation $\pi: V \mapsto V$ the prism $\pi G$ of $G$ is defined as follows: $V(\pi G)=V(G) \cup V\left(G^{\prime}\right)$, where $G^{\prime}$ is a copy of $G$, and $E(\pi G)=E(G) \cup E\left(G^{\prime}\right) \cup M_{\pi}$, where $M_{\pi}=\left\{u v^{\prime}: u \in V(G), v=\pi(u)\right\}$ and $v^{\prime}$ denotes the copy of $v$ in $G^{\prime}$.

We study and compare the properties of convex and weakly convex dominating sets in prism graphs. In particular, we characterize prism $\gamma_{\text {con }}$-fixers and -doublers. We also show that the differences $\gamma_{w c o n}(G)-\gamma_{w c o n}(\pi G)$ and $\gamma_{w c o n}(\pi G)-2 \gamma_{w c o n}(G)$ can be arbitrarily large, and that the convex domination number of $\pi G$ cannot be bounded in terms of $\gamma_{c o n}(G)$.
\end{abstract}

Keywords: domination, prism graphs.

2010 Mathematics Subject Classification: 05C69.

\section{REFERENCES}

[1] A.P. Burger, C.M. Mynhardt and W.D. Weakley, On the domination number of prisms of graphs, Discuss. Math. Graph Theory 2 (2004) 303-318. doi:10.7151/dmgt.1233

[2] G. Chartrand and F. Harary, Planar permutation graphs, Ann. Inst. Henri Poincare 3 (1967) 433-438.

[3] M. Lemańska and R. Zuazua, Convex universal fixers, Discuss. Math. Graph Theory 32 (2012) 807-812. doi:10.7151/dmgt.1631 
[4] C.M. Mynhardt and Z. Xu, Domination in prisms of graphs: universal fixers, Util. Math. 78 (2009) 185-201.

[5] M. Rosicka, A proof of the universal fixer conjecture, Util. Math., to appear.

[6] K. Wash, Edgeless graphs are the only universal fixers, Czechoslovak Math. J. 64 (2014) 833-843.

doi:10.1007/s10587-014-0136-3

Received 24 November 2017

Revised 3 September 2018

Accepted 3 September 2018 